\title{
Exploitation of gene knockout mice models to study the pathogenesis of leprosy
}

\author{
J. KRAHENBUHL \& L. B. ADAMS \\ National Hansen's Disease Programs Laboratory, Louisiana State \\ University School of Veterinary Medicine, Baton Rouge, Louisiana, \\ USA
}

Summary Shepard's technique for growth of Mycobacterium leprae in the mouse footpad, described in 1960, and more recent studies in thymectomized-irradiated mice and rats, athymic nude mice, nude rats and severe combined immunodeficiency (SCID) mice have defined the role of T-cell mediated immunity (CMI) in leprosy. However, the normal mouse and the immunocompromised mouse and rat represent only elements of polar tuberculoid disease and polar lepromatous leprosy, respectively. Transgenic, knockout (KO) mice may be employed to study the roles of individual genes in the ability of the host to mount an effective immune response to pathogens, and may also allow development of mouse models for the immunologically unstable borderline areas of the spectrum. We are exploiting certain $\mathrm{KO}$ mice to improve our understanding of CMI to M. leprae, and to study the role of the microenvironment of the leprosy granuloma in pathogenesis. CGD (chronic granulomatous disease) mice and iNOS-KO mice lack the ability to produce reactive oxygen intermediates (ROI) and reactive nitrogen intermediates (RNI), respectively, whereas the T cells of GKO mice are unable to produce interferon- $\gamma$ (IFN $\gamma$ ). iNOSKO mice exhibit an enhanced capacity to form granulomas, and the histopathology of the infected footpad tissues of this strain share many elements of borderline tuberculoid disease. The macrophages of CGD mouse kill or inhibit multiplication of $M$. leprae, although they lack ROI. Multiplication of the organisms in the footpad is enhanced in GKO mice, although these mice retain some host resistance. In addition, we have been investigating supplementary, conditional approaches to KO mouse models. For example, the down-regulatory effects of local prostaglandin production can be controlled with essential fatty acid deficient diets or indomethacin, RNI can be blocked in CGD and GKO mice by treatment with aminoguanidine, $\mathrm{N}^{\mathrm{G}}$ monomethyl arginine or $\mathrm{N}^{6}$-(1-iminoethyl)-L-lysine, and local elaboration of TNF $\alpha$ can be neutralized by anti-TNF $\alpha$ antibody or excess TNF $\alpha$ receptor. Other cytokines can be neutralized by antibody as well, broadening the range of conditional knockout models.

\section{Introduction}

Shepard's mouse footpad technique for cultivation of Mycobacterium leprae, described in $1960,{ }^{1}$ ranks as a milestone in leprosy research. The technique permitted isolation of organisms from lesions, discovery of new drugs, documentation of drug resistance and fundamental immunological studies. Soon thereafter, investigators applied this technique to 
immunosuppressed mice and rats, with the aim of developing models more representative of human leprosy. Work in thymectomized-irradiated mice, ${ }^{2}$ neonatally thymectomized rats, ${ }^{3}$ athymic (nude) mice, ${ }^{4}$ nude rats ${ }^{5}$ and severe combined immunodeficiency (SCID) mice ${ }^{6}$ helped define the role of T-cell mediated immunity (CMI) in leprosy, permitted more detailed evaluation of drug-regimens and allowed detection of 'persisting,' drug-susceptible $M$. leprae. The $M$. leprae-infected nude mouse has also provided a less expensive source of viable bacilli as a research reagent than did the armadillo.

However, these animal models represent only poor simulations of human leprosy. ${ }^{7}$ At best, the immunocompetent mouse and the immunocompromised mouse and rat feature only elements of polar tuberculoid disease and polar lepromatous leprosy, respectively. Because leprosy represents a fascinating model of human immunoregulatory disease, the development of murine models more representative of human leprosy is a research goal worthy of pursuit. Especially worthwhile would be a model of the broad borderline area of the spectrum, in which immunological instability often results in type 1 and 2 reactions. Understanding the basic mechanisms could lead to means of preventing or predicting these reactions, or of identifying the key components of CMI that need to be stimulated with an effective vaccine.

In recent years, exploitation of transgenic and knockout $(\mathrm{KO})$ strains of mice has shed light on the role of individual genes in effective CMI responses to a variety of pathogens. Our laboratory is exploiting certain $\mathrm{KO}$ strains to explore CMI to M. leprae both in vivo and in vitro, and to study the role of the microenvironment in leprosy granuloma in pathogenesis.

\section{Materials and methods}

Because of the expense and tedious nature of work with $M$. leprae in the mouse footpad, we narrowed our choice of KO models for our initial studies to a few that are relevant to our interest-the relationship between the M. leprae and its preferred host cell, the mononuclear phagocyte or macrophage $(\mathrm{MN}){ }^{8}$ Our initial studies are concerned with $\mathrm{KO}$ mice that lack genes important in key MN antimicrobial functions. CGD mice have a non-functional allele for the $\mathrm{gp}^{91 \text { phox }}$ subunit of phagocyte oxidase cytochrome $\mathrm{b}$, and serve as a model of chronic granulomatous disease. ${ }^{9}$ The polymorphonuclear leukocytes and $\mathrm{MN}$ of $\mathrm{CGD}$ mice cannot produce reactive oxygen intermediates (ROI), hydrogen peroxide, superoxide anion, or hydroxyl radical. ${ }^{10}$ GKO mice cannot produce interferon- $\gamma($ IFN $\gamma)$, the primary MN activating factor. ${ }^{10}$ iNOS KO mice have a targeted disruption in the calmodulin binding-site of the inducible nitric oxide synthase (iNOS) gene. ${ }^{11} \mathrm{MN}$ from iNOS $\mathrm{KO}$ mice cannot produce reactive nitrogen intermediates (RNI), chiefly the L-arginine-dependent production of nitric oxide, an RNI that represents a potent antimicrobial mechanism directed at intracellular M. leprae by activated $\mathrm{MN}$. $^{12}$

Studies were carried out with each of these strains, in which the multiplication of $M$. leprae in the footpads was examined over extended periods. Development of granuloma was monitored by histopathological and, in some cases, immunohistological changes and cytokine response. Finally, the ability of $\mathrm{MN}$ to cope with $M$. leprae in vitro was evaluated in these $\mathrm{KO}$ strains. 


\section{Results}

CGD MICE

Our previous studies ${ }^{13}$ employing CGD mice demonstrated that peritoneal and bone marrow MN were unable to produce ROI when stimulated with $\operatorname{IFN} \gamma$, although they did produce normal amounts of RNI. Activated MN obtained from CGD mice were fully competent in their ability to kill $M$. tuberculosis, although growth of the tubercle bacillus was markedly enhanced in the lungs of CGD mice in comparison to controls, and these mice failed to survive as long as the controls.

In vitro, CGD MN activated with IFN $\gamma$ produced RNI, and were able to kill or inhibit $M$. leprae. Over the 6-month course of footpad infection of CGD mice, the growth of M. leprae was not different from that of controls, reaching plateau between 4 and 6 months after inoculation. Whereas the number of $M$. leprae gradually declined in control mice over the subsequent 6-9 months, long term evaluation of the CGD mice was not possible, because all but one mouse failed to survive beyond 6 months after infection. Studies in CGD mice are continuing both in vivo and in vitro.

GKO MICE

Previous studies of $M$. tuberculosis infection of GKO mice ${ }^{14}$ revealed that they were highly susceptible to infection. Preliminary studies have showed that the maximum of multiplication of $M$. leprae in the footpads of GKO mice was approximately 10 -fold greater than that in the controls, and was achieved about 5 months after inoculation, 1 month later than in the controls; however, the numbers of $M$. leprae per footpad of GKO mice paralleled those in control mice thereafter. Interestingly, the granulomatous response in the $M$. leprae-infected GKO footpads was greatly enhanced. The cellular infiltrate, composed of epithelioid MN and scattered lymphocytes, was not organized into well-formed granulomas, but replaced as much as $75 \%$ of the footpad tissue. Although GKO mice cannot produce IFN $\gamma$, GKO mouse $\mathrm{MN}$ could be activated to kill or inhibit $M$. leprae when they were exposed to IFN $\gamma$. Enhanced production of RNI was seen in these activated MN. Moreover, T-cell blast transformation in response to soluble $M$. leprae antigen in vitro was greatly enhanced in cells obtained from the draining lymph node cells of GKO mice. These studies are continuing.

iNOS KO MICE

As we have shown, ${ }^{13,15}$ IFN $\gamma$-activated MN obtained from iNOS KO mice produced normal amounts of ROI, but failed to produce RNI. In contrast to GCD and GKO MN, IFN $\gamma$ activated iNOS KO MN could not cope with M. tuberculosis in vitro. In vivo, iNOS KO mice were more susceptible than were controls to infection with $M$. tuberculosis. ${ }^{13}$ Growth of the organisms was enhanced in the spleen and lungs, and iNOS mice succumbed after 3-4 months to an intravenous infecting dose of M. tuberculosis that failed to kill any of the controls in the course of 12 months.

In the case of $M$. leprae infection of the footpad, the organism multiplied to slightly higher plateau values in iNOS KO mice than in controls. However, in terms of the number of organisms per footpad, the subsequent course of the infection was indistinguishable from that in control mice, the number of organisms decreasing gradually by more than $90 \%$ in the course of the subsequent 7-12 months. On the other hand, marked differences of local 
granuloma formation were observed in the footpads of iNOS KO mice. Whereas infected control mice demonstrated small focal collections of mononuclear cells immediately beneath the epidermis, the footpads of the infected iNOS KO mice were clearly indurated; granulomatous inflammation in the iNOS KO foot pads, which occupied more than 10 times the volume of that in the controls, consisted of dense organized collections of lymphocytes and epithelioid MN infiltrating the neuromuscular bundles, and resembled elements of human borderline tuberculoid disease. Grossly, the feet of infected iNOS KO mice were visibly enlarged in comparison to those of the control infected mice, in which no gross changes were observed.

Following a larger innoculum $\left(10^{8}\right)$ of $M$. leprae into the footpads, the feet of iNOS KO mice became obviously indurated. Enhanced granuloma formation was also apparent in the liver tissue of iNOS KO mice that had been infected intravenously with $M$. leprae. In this organ, highly organized granulomas consisting of lymphocytes, epithelioid MN and multinucleated giant cells were both more numerous and markedly larger than in the poorly organized granulomas of the controls. Immunohistological staining revealed more numerous CD4+ $\mathrm{T}$ cells in iNOS KO granulomas, and a tendency for CD8+ cells to localize at the periphery of the granulomas, as has been shown in human TT/BT disease. ${ }^{16}$

\section{Discussion}

A study of the course of $M$. leprae infection in $\mathrm{KO}$ mouse models should advance our understanding of host resistance to the organism. Selection of the appropriate KO strain must be made carefully, as evaluation of the multiplication of $M$. leprae and granuloma formation in the footpad requires prolonged observation. However, during the course of these extended studies, results can be obtained in the shorter term from ex vivo or in vitro experiments carried out with purified cell populations, or in situ, using PCR to examine cytokine profiles.

Our current work is focused on $\mathrm{KO}$ models, in which the targeted gene deletions are important in $\mathrm{MN}$-mediated anti- $M$. leprae effector functions. We intend also to explore $\mathrm{KO}$ strains lacking the CD4 and CD8 T cell subsets and, in addition to GKO mice, KO strains in which the deleted genes control production of cytokines relevant to the TH1/TH2 paradigm.

The findings from our studies in CGD and iNOS KO mice, which compared the relative contributions of the ROI and RNI pathways, showed clearly that the RNI system was more critical than the ROI system in activated MN-mediated anti- $M$. leprae function. However, it was also clear from our studies in the GKO and iNOS KO models that host resistance to $M$. leprae was not ablated by the deletion of such potent single pathways as production of IFN $\gamma$ or iNOS, showing clearly that the mechanism of CMI to infection does not fit any simple unitary hypothesis. In general, the iNOS-KO and GKO mice displayed an enhanced capacity to form granulomas as an apparent, compensatory mechanism to their respective lack of distinct, powerful MN anti-microbial functions.

Our data also suggested that the use of $M$. leprae for study in $\mathrm{KO}$ mice might represent a unique opportunity to dissect the mechanisms of CMI, especially those important in human leprosy, in a manner not possible with other organisms such as $M$. tuberculosis. The relevance of animal models to human tuberculosis has been long debated. ${ }^{17}$ The finding of greatly enhanced growth and increased mortality in $M$. tuberculosis-infected iNOS $\mathrm{KO}^{13,18}$ and GKO mice ${ }^{14}$ underscores the importance of these primary defence mechanisms, but the virulence of M. tuberculosis infection of the mouse appears likely to have overshadowed a number of 
intact, redundant mechanisms of resistance remaining in these $\mathrm{KO}$ mice that may also be important in human tuberculosis. By contrast, the slow growth, low virulence and lack of toxicity of $M$. leprae permit the exploration of redundant or contingent mechanisms of resistance, the very mechanisms that may be relevant to the unstable nature of the borderline stage in leprosy.

It is our intention to explore other $\mathrm{KO}$ models as well. However, we are currently seeking to determine if our current studies lend themselves to additional 'conditional' KO approaches. The down-regulatory effects of local prostaglandin production, which can be controlled in each model by essential fatty acid-deficient diets or indomethacin, are highly relevant to $\mathrm{CMI}$ in the microenvironment of the leprosy granuloma. ${ }^{19}$ Treatment of mice with aminoguanidine, ${ }^{13,20}, \mathrm{~N}^{\mathrm{G}}$ monomethyl arginine ${ }^{21}$ or $\mathrm{N}^{6}$-(1-iminoethyl)-L-lysine can block RNI and, in CGD or GKO mice, provide a 'conditional KO' approach. Similarly, local elaboration of TNF $\alpha$ can be neutralized by anti-TNF $\alpha$ antibody ${ }^{22}$ or, as we have shown, with excess TNF $\alpha$ receptor. ${ }^{23}$ Obviously, other selected cytokines can be neutralized as well to ablate other host-defence pathways.

Is there a single $\mathrm{KO}$ mouse model that will permit examination of the unique immunopathological spectrum of human leprosy, especially the unstable borderline area of the spectrum? Probably not. Can KO mouse models give insight into the mechanisms of relapse or leprosy reactions? Clearly, yes. The iNOS-KO mice demonstrated an enhanced capacity to form granulomas, apparently as a means of compensating for their lack of $\mathrm{MN}$ antimicrobial function; these granulomas displayed key histological features of borderline tuberculoid disease.

In addition to enhancing our understanding of mechanisms of resistance, continued examination of the course of infection by $M$. leprae and detailed immunohistological and cytokine profile analysis in these and other $\mathrm{KO}$ models may lead to a means of predicting or preventing type 1 or 2 reactions.

\section{References}

${ }^{1}$ Shepard CC. The experimental disease that follows the injection of human leprosy bacilli into footpads of mice. J Exp Med, 1960; 112: 445-454.

2 Rees RJW. Enhanced susceptibility of thymectomised and irradiated mice to infection with M. leprae. Nature, 1968; 211: 657-658.

${ }^{3}$ Fieldsteel AH, McIntosh J. Effect of neonatal thymectomy and anti-lymphocyte serum on susceptibility of rats to M. leprae. Proc Soc Exp Biol Med, 1971; 138: 408-413.

${ }^{4}$ Colston MJ, Kohsaka K. The nude mouse in studies of leprosy. In: Fogh J, Giovanella BC (eds) The nude mouse in experimental and clinical research, vol. 2, Academic Press, New York, 1982, pp 247-266.

5 Dawson P, Colston J, Fieldsteel AH. Infection of the congenitally athymic rat with Mycobacterium leprae. Int J Lepr, 1983; 51: 336-346.

6 Yogi Y, Nakamura K, Inoue T et al. Susceptibility of severe combined immunodeficient mice to Mycobacterium leprae: multiplication of the bacillus and dissemination of the infection at early stage. Jpn J Lepr, 1991; 60: 139145.

7 Jacobson RR, Krahenbuhl JL. Leprosy. Lancet, 1999; 353: 655-660.

8 Krahenbuhl JL, Adams LB. The interaction between the macrophage and the leprosy bacillus. In: Zwilling B, Eisenstein T (eds) Macrophage-pathogen interactions. Marcel-Dekker, New York, 1993, pp 281-302.

9 Morgenstern DE, Gifford MAC, Li LL et al. Absence of respiratory burst in X-linked chronic granulomatous disease mice leads to abnormalities in both host defense and inflammatory response to Aspergillus fumigatus. J Exp Med, 1997; 185: 207-218.

10 Ding AH, Nathan CF, Stuehr DJ. Release of reactive nitrogen intermediates and reactive oxygen intermediates from mouse peritoneal macrophages. Comparison of activating cytokines and evidence for independent production. J Immunol, 1988; 141: 2407-2412. 
11 Laubach VE, Shesely EG, Smithies O, Sherman PA. Mice lacking inducible nitric oxide synthase are not resistant to lipopolysaccharide-induced death. Proc Natl Acad Sci, 1995; 92: 10688-10692.

12 Adams LB, Franzblau SG, Vavrin Z et al. L-arginine dependent macrophage effector functions inhibit metabolic activity of Mycobacterium leprae. J Immunol, 1991; 147: 1642-1646.

13 Adams LB, Dinauer MC, Morgenstern DE, Krahenbuhl JL. Comparison of the roles of reactive oxygen and nitrogen intermediates in the host response to Mycobacterium tuberculosis using transgenic mice. Tubercle Lung Dis, 1997; 78: 237-246.

14 Cooper AM, Dalton DK, Stewart TA et al. Disseminated tuberculosis in interferon- $\gamma$ gene disrupted mice. $J$ Exp Med, 1993; 178: 2243-2247.

15 Adams LB, Job CK, Krahenbuhl J. Role of inducible nitric oxide synthase in resistance to Mycobacterium leprae in mice. Infect Immun, 2000, in press.

16 Modlin RL, Melancon-Kaplan J, Young SMM, Rea T. Learning from lesions: patterns of tissue inflammation in leprosy. Proc Natl Acad Sci USA, 1988; 85: 1213-1217.

17 Orme I, Collins F. Animal models of tuberculosis. In: Bloom B (ed) Tuberculosis, pathogenesis, protection and control. ASM Press, Washington, 1994, pp 113-134.

${ }^{18}$ MacMicking J, North RJ, LaCourse R et al. Identification of nitric oxide synthase as a protective locus against tuberculosis. Proc Natl Acad Sci USA, 1997; 94: 5243-5248.

19 Adams LB, Gillis TP, Hwang HD, Krahenbuhl JL. Effects of essential fatty acid deficiency on PGE $_{2}$ production and cell mediated immunity in a mouse model of leprosy. Infect. Immun, 1997; 65: 1152-1157.

${ }^{20}$ Chan J, Tanaka K, Carroll P et al. Effects of nitric oxide synthase inhibitors on murine infection with Mycobacterium tuberculosis. Infect Immun, 1995; 63: 736-740.

21 Granger DL, Hibbs JB. High-output nitric oxide: weapon against infection? Trends Microbiol, 1996; 4: 46-47.

22 Kindler V, Sappino AP, Grau GE et al. The inducing role of tumor necrosis factor in the development of bacterial granulomas during BCG infection. Cell, 1989; 56: 731-740.

23 Adams LB, Mason CM, Kolls JK et al. Exacerbation of acute and chronic murine tuberculosis by administration of a TNF receptor-expressing adenovirus. J Infect Dis, 1995; 171: 400-405. 